\title{
DETECTION OF A COMPACT NUCLEAR RADIO SOURCE IN THE LOCAL GROUP ELLIPTICAL GALAXY M32
}

\author{
Yang Yang ${ }^{1,2,3}$, Zhiyuan $\mathrm{Li}^{1,2,3}$, Loránt O. Sjouwerman ${ }^{4}$, Q. Daniel Wang ${ }^{1,5}$, Qiusheng Gu ${ }^{1,2,3}$, \\ Ralph P. Kraft ${ }^{6}$, Feng Yuan ${ }^{7}$ \\ lizy@nju.edu.cn
}

\begin{abstract}
The Local Group compact elliptical galaxy M32 hosts one of the nearest candidate super-massive black holes (SMBHs), which has a previously suggested X-ray counterpart. Based on sensitive observations taken with the Karl G. Jansky Very Large Array (VLA), we detect for the first time a compact radio source coincident with the nucleus of M32, which exhibits an integrated flux density of $\sim 47.3 \pm 6.1 \mu \mathrm{Jy}$ at $6.6 \mathrm{GHz}$. We discuss several possibilities for the nature of this source, favoring an origin of the long-sought radio emission from the central $\mathrm{SMBH}$, for which we also revisit the X-ray properties based on recently acquired Chandra and XMM-Newton data. Our VLA observations also discover radio emission from three previously known optical planetary nebulae in the inner region of M32.
\end{abstract}

Subject headings: galaxies: nuclei —galaxies: individual (M32)-radio continuum: galaxies

\section{Introduction}

It is recognized that super-massive black holes (SMBHs), commonly residing in the nuclei of present-day galaxies with a substantial stellar bulge, have grown the bulk of their mass during quasar phases, but otherwise spent the majority of their life accreting at rates well below the Eddington limit (e.g., Soltan 1982; Yu \& Tremaine 2002; Marconi et al. 2004). As such, most SMBHs

\footnotetext{
${ }^{1}$ School of Astronomy and Space Science, Nanjing University, Nanjing 210093, China

${ }^{2}$ Key Laboratory of Modern Astronomy and Astrophysics, Nanjing University, Nanjing 210093, China

${ }^{3}$ Collaborative Innovation Center of Modern Astronomy and Space Exploration, Nanjing 210093, China

${ }^{4}$ National Radio Astronomy Observatory, Socorro, NM 87801, USA

${ }^{5}$ Department of Astronomy, University of Massachusetts, Amherst, MA 01003, USA

${ }^{6}$ Harvard-Smithsonian Center for Astrophysics, 60 Garden Street, Cambridge, MA 02138, USA

${ }^{7}$ Shanghai Astronomical Observatory, Chinese Academy of Sciences, Shanghai 200030, China
} 
in the local Universe manifest themselves as low-luminosity active galactic nuclei (LLAGNs; see review by Ho 2008), the imprint of which is often difficult to discern. Nevertheless, dedicated surveys of nearby LLAGNs carried out in the past two decades have yielded high detection rates in the radio and X-ray bands (e.g., Nagar et al. 2000; Ho \& Ulvestad 2001; Gallo et al. 2010; Miller et al. 2012), providing important clues to their nature. The current consensus is that LLAGNs are powered by a radiatively inefficient, advection-dominated accretion flow (ADAF; Narayan \& Yi 1994) that operate at very sub-Eddington accretion rates, which is probably coupled with outflows in the form of jets and/or winds (see review by Yuan \& Narayan 2014).

In the Local Group, strong dynamical evidence of a SMBH have been found for all of the three galaxies with a prominent bulge: Milky Way, M31 and M32, among which our own Galaxy makes the most compelling case (Genzel, Eisenhauer \& Gillessen 2010). Interestingly, these three nearest SMBHs also show the lowest Eddington ratio 1 among known LLAGNs, thus supplying us with unique opportunities to explore the poorly understood physics of SMBHs at the most quiescent state.

In the compact elliptical galaxy M32, a central SMBH of $\sim 2.5 \times 10^{6} \mathrm{M}_{\odot}$ has been inferred from modeling of its circumnuclear stellar kinematics (Verolme et al. 2002; van den Bosch \& de Zeeuw 2010). Additional evidence for an accreting SMBH comes from the Chandra detection of an X-ray source coincident with the nucleus of M32 (Ho, Terashima \& Ulvestad 2003, hereafter HTU03), which exhibits a power-law spectrum with a remarkably low 2-10 keV luminosity of $\sim 10^{36} \mathrm{erg} \mathrm{s}^{-1}$, corresponding to only $\sim 10^{-8.5}$ of its Eddington luminosity. On the other hand, the SMBH leaves little, if any, signature at the optical and infrared bands, presumably owing to the dearth of interstellar medium (ISM) in M32 (e.g., Ho, Filippenko \& Sargent 1997; Sage, Welch \& Mitchell 1998; Welch \& Sage 2001; Revnivtsev et al. 2007; but see Seth 2010). Even at the seemingly more promising radio bands, where synchrotron radiation from a putative jet is expected, a firm detection in the literature has been absent. Compiling Very Large Array observations taken before 2000, HTU03 derived $5 \sigma$ upper limits of $875,30,525$ and $550 \mu \mathrm{Jy}$ at $15,8.4,5$ and $1.4 \mathrm{GHz}$, respectively.

In this work, we report the detection of a compact radio source at the nucleus of M32 based on high-resolution, sensitive observations recently carried out with the Karl G. Jansky Very Large Array (VLA). We also revisit the properties of the X-ray nucleus identified by HTU03, using recently acquired Chandra data XMM-Newton data. We adopt a distance of $780 \mathrm{kpc}$ for M32, effectively placing it at the same distance as M31 (Young et al. 2008). Throughout this work we quote $1 \sigma$ uncertainties unless otherwise stated. All coordinates are given for epoch J2000.

\footnotetext{
${ }^{1} \mathrm{Sgr} \mathrm{A}^{*}$ and $\mathrm{M} 31^{*}$ have an Eddington ratio of $L_{\mathrm{bol}} / L_{\mathrm{Edd}} \sim 10^{-8.5}$ (Baganoff et al. 2003; Li et al. 2011), where $L_{\mathrm{bol}}$ is the bolometric luminosity and $L_{\mathrm{Edd}} \equiv 1.3 \times 10^{38}\left[M_{\mathrm{BH}} / M_{\odot}\right] \mathrm{erg} \mathrm{s}^{-1}$ the Eddington luminosity, for masses of $\sim 4 \times 10^{6} \mathrm{M}_{\odot}$ (Ghez et al. 2008) and $\sim 1.4 \times 10^{8} \mathrm{M}_{\odot}$ (Bender et al. 2005), respectively. The present work infers $L_{\mathrm{bol}} / L_{\mathrm{Edd}} \sim 10^{-7.5}$ for the $\mathrm{SMBH}$ in $\mathrm{M} 32$.
} 


\section{Observations}

\subsection{Radio data}

Three VLA observations of M32 were carried out in the B-configuration on July 24, 28 and 29, 2012 (Program ID: 12A-243; PI: L. Sjouwerman). Taken in the $C$-band, each observation has a total bandwidth of $2 \mathrm{GHz}$ centered at $6.6 \mathrm{GHz}$ and an integration time of $6 \mathrm{hrs}$. The flux calibrator was $3 \mathrm{C} 48$ with a $3 \%$ systematic uncertainty (Perley \& Butler 2013). The observations were phase-referenced to the calibrator J0038+4137 every 8 minutes with a switching angle of 1.1. The positional accuracy of our phase calibrator was $\lesssim 0{ }^{\prime \prime} 002$. For each observation, the data was flagged, calibrated, and imaged following standard procedures with the Common Astronomy Software Applications 2 (CASA, version 3.4.0). We have used the SPLIT task to average the 64 channels of each spectral window with width $=2$, and employed the CLEAN task using the MultiFrequency Synthesis mode, nterms $=1$, and Natural weighting to achieve maximum sensitivity. The resultant images were made for a primary beam of $\sim 380^{\prime \prime}$ and sampled the sysnthesized beam of $1^{\prime \prime} .14 \times 1^{\prime \prime}$. 09 with $0^{\prime \prime} 3$ pixels. We have also used the CONCAT task to concatenate the three visibility data sets to produce the final, most sensitive image (Figure 1a), which has an average rms noise of $1.2 \mu \mathrm{Jy}_{\mathrm{beam}^{-1}}$. Notably, this is a factor of $\sim 90(5)$ lower than that achieved by HTU03 at the neighboring frequency of $5(8.4) \mathrm{GHz}$.

\subsection{X-ray data}

The field of M32 has been observed by Chandra with its Advanced CCD Imaging Spectrometer (ACIS) on nine epochs between 2000-2005. For each of these observations, the raw data was downloaded from the public archive and reprocessed following the standard procedure, using CIAO v4.3 and the corresponding calibration files. The relative astrometry among the individual observations was calibrated by matching centroids of point-like sources commonly detected in the overlapping field of view. In addition, we have carried out deep XMM-Newton observations of M32 (PI: Q.D. Wang) on four epochs in 2011, with a total exposure of $\sim 400 \mathrm{ks}$. For each observation, the data obtained from the European Photon Imaging Camera (EPIC) was reduced following the standard procedure, using SAS v11.0.1 and the corresponding calibration files. Table 1 gives a log of the X-ray data.

\footnotetext{
${ }^{2}$ http://casa.nrao.edu
} 


\section{Analysis and results}

\subsection{Detection of radio sources}

Figure 17 displays the inner $25^{\prime \prime} \times 25^{\prime \prime}$ region of M32 in the concatenated $C$-band image, overlaid by optical intensity contours (cyan) that trace the starlight. A compact source, which we designate $\mathrm{R} 1$, is clearly present at the optical center of M32. R1 shows a peak flux density $\sim 10.7 \pm 1.2$ $\mu \mathrm{Jy}$ beam $^{-1}$, but it appears more extended than a point source. Thus we performed a single elliptical Gaussian fit to R1 using the CASA task IMFIT. The fit suggested that the source is formally larger than the synthesized beam, with a deconvolved FWHM $\approx[(2$ "! $8 \pm 0$ '! 2$) \times(1$ !! $8 \pm$ $\left.\left.0^{\prime \prime} 2\right)\right](\sim 10.6 \mathrm{pc} \times 6.8 \mathrm{pc})$ and a positional angle of $130.1 \pm 1.9$. The peak of R1 was found at $[\mathrm{RA}, \mathrm{DEC}]=\left[00^{h} 42^{m} 41^{\mathrm{s}} .838 \pm 0.007,+40^{\circ} 51^{\prime} 54^{\prime \prime} .89 \pm 0^{\prime \prime} \cdot 07\right]$, which is coincident with the 2MASS position of the nucleus, $\left[00^{h} 42^{m} 41^{\mathrm{s}} .825,+40^{\circ} 51^{\prime} 54^{\prime \prime} .61\right]$, to within the uncertainty of the latter $\left(\lesssim 0^{\prime \prime} 5\right.$; Jarrett et al. 2003). An integrated flux density of $47.3 \pm 6.1 \mu \mathrm{Jy}$ was measured for R1 in the concatenated image. The uncertainty is the quadratic sum of the error reported by IMFIT and the $3 \%$ uncertainty in the absolute flux scale. We also measured the integrated flux density of R1 in the individual observations, but found no statistically significant variation. We further divided the full bandwidth into two 1-GHz sub-bands centering at 6 and $7 \mathrm{GHz}$ to measure the spectral index, $\alpha$, defined as $\mathrm{S}_{\nu} \propto \nu^{\alpha}$. The result was only poorly-constrained, with $\alpha \approx-2.0 \pm 1.7$.

Three faint, off-nucleus sources are also evident in Figure 1a, which we designate as R2, R3 and R4. They all lie within a projected distance of $10^{\prime \prime}$ from the nucleus and have a significance of $\sim 3 \sigma$ in the concatenated image. Table 2 gives their positions and flux densities. Interestingly, all three sources are spatially coincident with planetary nebula (PNe) identified in the SAURON integral field spectroscopic observations of M32 (Sarzi et al. 2011; their PNe \#5, \#3 and \#7 are coincident with R2, R3 and R4, respectively). This is demonstrated by contrasting the white and cyan intensity contours in Figure 1a. The latter are derived from an archival Hubble Space Telescope (HST) WFC/F502N image (Program ID: 11714). At the position of each off-nuclear radio source, a compact F502N source, presumably dominated by the [O III $] \lambda 5007$ line emission, is clearly seen. Therefore, we suggest that the three off-nucleus sources are radio counterparts of known PNe in M32, which most likely arise from free-free emission (Kwok 2000). To our knowledge, this signifies the first detection of radio PNe at the far-side of the Local Group. The 6.6 GHz monochromatic luminosities 3 of the three sources, $\sim 2 \times 10^{31} \mathrm{erg} \mathrm{s}^{-1}$, are comparable to NGC 7027, one of the most luminous Galactic PNe with a $4.9 \mathrm{GHz}$ monochromatic luminosity of $\sim 3 \times 10^{31} \mathrm{erg} \mathrm{s}^{-1}$ (Zijlstra, van Hoof \& Perlev 2008).

\footnotetext{
${ }^{3} \mathrm{~L}_{R} \equiv \nu \mathrm{L}_{\nu}$
} 


\section{2. $\quad$ Revisiting the $\mathrm{X}$-ray nucleus}

Figure 1 $\mathrm{b}$ shows a $0.3-8 \mathrm{keV}$ image for the same region as in Figure 1a, obtained by combining the nine Chandra/ACIS observations. Three discrete sources, which have been previously designated as X1, X2 and X3 by HTU03, are clearly present, and they remain the only sources detected within this region, despite the $\sim 3$ times deeper total exposure achieved here. Among them, X1 is coincident with the nucleus and has been suggested to be the X-ray counterpart of the SMBH by HTU03. The slight offset $\left(\sim 00^{\prime \prime} 34\right)$ between the centriod of X1 and the nucleus in the WFC/F502N image is consistent with the astrometry accuracy 4 . From each ACIS observation, we extracted a spectrum for X1 from a $2^{\prime \prime}$-radius circle, and a background spectrum from an annulus with innerto-outer radii of $2^{\prime \prime}-4^{\prime \prime}$. In the following we restrict our analysis to energies above $0.5 \mathrm{keV}$, so that contamination from X2, the nearby very soft source, is negligible. We fitted the individual spectra using an absorbed power-law model (wabs ${ }^{*}$ powerlaw in XSPEC), fixing the equivalent hydrogen column density at the Galactic foreground value, $7 \times 10^{20} \mathrm{~cm}^{-2}$. This is justified by the fact that M32 exhibits no detectable neutral ISM (Welch \& Sage 2001). For ObsIDs 2017, 2494 and 5690, which have good signal-to-noise ratios, we were able to constrain the photon-index, $\Gamma$, to be $2.50_{-0.17}^{+0.17}$, $2.72_{-0.18}^{+0.21}$ and $2.36_{-0.10}^{+0.09}$, respectively. For the remaining ObsIDs, we fixed the photon-index at 2.4 and constrained the normalization only. The spectral models were then used to predict the 0.5-10 $\mathrm{keV}$ intrinsic luminosity of $\mathrm{X} 1$ at each epoch.

The moderate angular resolution afforded by the XMM-Newton/EPIC observations is not optimal for isolating the emission from X1, given the presence of the brighter source X3 located $\sim 9^{\prime \prime}$ away (Figure 1b). Therefore, we employed a two-dimensional image fitting method (Li et al. 2011) to simultaneously determine the observed fluxes of X1 and X3, which not only maximizes the counting statistics, but also accounts for the propagation of the dominating error arising from the mutual PSF scattering. Briefly, we fitted the $0.5-8 \mathrm{keV}$ image of the central $\sim 60^{\prime \prime} \times 60^{\prime \prime}$ field with a three-component model convolved with the PSF. The model consists of a constant local background and two point sources (for X1 and X3), each represented by a delta function. We mimicked the PSF using the image of a bright transient source recently found in the bulge of M31 (Kaur et al. 2012), averaged over four XMM-Newton observations (ObsIDs 0600660301, 0600660401, 0600660501 and 0600660601). We jointly fitted the EPIC MOS1 and MOS2 images taking into account their distinct PSFs. We did not include data from the PN due to its poorer angular resolution. In the fit, we fixed the relative offset between X1 and X3, as determined from the combined Chandra/ACIS image. The best-fit was obtained by minimizing the C-statistic (Cash et al. 1979). The resultant count rates and uncertainties of $\mathrm{X} 1$ from the individual observations were then converted into an intrinsic luminosity by adopting the above absorbed power-law spectral model $(\Gamma=2.4)$. We have added to the error budge a $10 \%$ uncertainty in quadrature, to account for the uncertainty in the adopted photon-index. A consistency check indicates that the average flux ratio between $\mathrm{X} 1$ and $\mathrm{X} 3$ is in

\footnotetext{
${ }^{4}$ The typical pointing accuracy of both HST and Chandra is $\lesssim 1$ ". However, owing to the lack of common sources, it is virtually impossible to match the optical/X-ray astrometry to better than the individual pointing accuracy.
} 
good agreement with that measured from the Chandra observations.

The 0.5-10 keV intrinsic luminosity and its uncertainty thus determined for each observation are given in the last column of Table 1. Figure 2 shows the long-term light curve of X1 over $\sim 11$ yrs. The average luminosity seems to have increased by a factor of $\sim 2$ since 2002 , although the sparse temporal sampling could have missed intervals of substantially weaker or stronger radiation. In addition, the luminosity was seen to increase by $\sim(190 \pm 100) \%$ between two XMM-Newton observations separated by two days (Table 1). We also searched for but found no significant intraobservation flux variation in either the Chandra or XMM-Newton exposures.

\section{Discussion and summary}

The VLA observations with unprecedented sensitivity have helped us establish the presence a compact radio source in the nucleus of M32. The source, R1, has a monochromatic luminosity of $\sim 2.3 \times 10^{32} \mathrm{erg} \mathrm{s}^{-1}$ at $6.6 \mathrm{GHz}$. This remarkably weak emission and the lack of significant variability hinder a straightforward identification of its nature. We discuss several possibilities below and favor an association with the putative SMBH.

First, R1 can in principle arise from by synchrotron or free-free emission powered by, for instance, nuclear star-forming activities, in which case the source might span a physical size of $\sim 10 \mathrm{pc}$, as its slightly extended morphology suggests (Section 3.1). However, there is virtually no evidence for recent or on-going star formation at the center of M32 - in fact, none across the entire

galaxy (Brown et al. 2000), disfavoring the presence of any recent core-collasped supernovae. The expected rate of Type Ia supernovae at the nucleus is also very low $\left(\lesssim 10^{-8} \mathrm{yr}^{-1}\right.$; HTU03). Thus, $\mathrm{R} 1$ is unlikely to be associated with synchrotron emission from a supernova remnant. Neither is there evidence for a circumnuclear ionized gas in M32. Ho et al. (2003) reported a $3 \sigma$ upper limit in the $\mathrm{H} \alpha$ luminosity of $\lesssim 2 \times 10^{36} \mathrm{erg} \mathrm{s}^{-1}$. Assuming a typical electron temperature $T_{e} \approx 10^{4} \mathrm{~K}$, we can use the following relation to estimate the strength of free-free emission from any circumnuclear ionized gas (Caplan \& Deharveng 1986):

$$
\left[\frac{F(\mathrm{H} \alpha)}{10^{-12} \mathrm{erg} \mathrm{cm}^{-2} \mathrm{~s}^{-1}}\right] \sim 0.8\left(\frac{T_{e}}{10^{4} \mathrm{~K}}\right)^{-0.52}\left(\frac{\nu}{\mathrm{GHz}}\right)^{0.1}\left(\frac{S_{\mathrm{ff}}}{\mathrm{mJy}}\right) .
$$

The above upper limit in $\mathrm{H} \alpha$ luminosity corresponds to $S_{\mathrm{ff}, 6.6 \mathrm{GHz}}<28.4 \mu \mathrm{Jy}$. This is significantly lower than the flux density of R1, suggesting that the bulk of the observed radio emission does not come from free-free emission.

Alternatively, we might have caught a transient radio source similar to those found in the center of our Galaxy (e.g., Davies et al. 1976; Zhao, Roberts \& Goss 1992; Hyman et al. 2002, 2009). In particular, the Galactic Center Transient (GCT) detected at a projected distance of $\sim 1.4$ pc from Sgr A* during 1990-1991 would have a $6.6 \mathrm{GHz}$ flux density of $\sim 10-20 \mu \mathrm{Jy}$ if located at the distance of M32 (Zhao et al. 1992). The nature of some of these transients, including the GCT, remains 
uncertain. One favored suggestion was that they are radio outbursts of black hole binaries (BHBs), although in most cases the expected X-ray emission was actually not detected. R1 could be such a radio transient, provided that it was not physically related to the stable source X1.

Can, then, both $\mathrm{R} 1$ and $\mathrm{X} 1$ be the persistent counterpart of a BHB located in the center of M32? Indeed, the identification of X1 as the X-ray counterpart of the SMBH essentially rested on positional coincidence (HTU03). The very modest luminosity of $\mathrm{X} 1, \sim 10^{36} \mathrm{erg} \mathrm{s}^{-1}$, lies within the range of X-ray binaries (XRBs) in the low/hard state. The Chandra and XMM-Newton observations now suggest that X1 has persisted over a decade (Section 3.2), hence it is not an X-ray transient similar to those found in the Galactic center, which are likely outbursting BHBs (Muno et al. 2005). Moreover, its steep power-law spectrum $(\Gamma \sim 2.4)$ is atypical of XRBs in the low/hard state, which generally exhibit $\Gamma \sim 1.5-2$ (e.g., Remillard \& McClintock 2006, Wu \& Gu 2008). Furthermore, the BHB case is strongly disfavored on the basis of the "fundamental plane of black hole activity" (hereafter the FP; Merloni. Heinz \& di Matteo 2003; Falcke. Körding \& Markoff 2004), an empirical relation linking the radio luminosity $\left(L_{\mathrm{R}}\right.$, practically evaluated at $\left.5 \mathrm{GHz}\right)$, X-ray luminosity ( $L_{\mathrm{X}}$, evaluated as $\left.L_{2-10 \mathrm{keV}}\right)$ and black hole mass $\left(M_{\mathrm{BH}}\right)$, in the form of Gültekin et al. (2009):

$$
\log L_{\mathrm{R}}=(4.80 \pm 0.24)+(0.78 \pm 0.27) \log M_{\mathrm{BH}}+(0.67 \pm 0.12) \log L_{\mathrm{X}}
$$

Following this relation, a BHB with $M_{\mathrm{BH}} \sim 10 M_{\odot}$ and $L_{2-10 \mathrm{keV}} \sim 10^{36} \mathrm{erg} \mathrm{s}^{-1}$, is expected to have $L_{5} \mathrm{GHz}$ of $\sim 5 \times 10^{29} \mathrm{erg} \mathrm{s}^{-1}$. This is $\sim 400$ times lower than our measurement for R1, when extrapolated from the neighboring frequency of $6.6 \mathrm{GHz}$ assuming a canonical spectral index of $\alpha=-0.7$.

Lastly, R1 may be the long-sought radio counterpart of the central SMBH, which most likely arises from radio synchrotron radiation. If this were the case, $\mathrm{R} 1$ would be one of the weakest known "AGNs" in radio, along with $\operatorname{Sgr} A^{*}\left(L_{5 \mathrm{GHz}} \sim 3 \times 10^{32} \mathrm{erg} \mathrm{s}^{-1}\right.$; Zhao, Bower \& Goss 2001) and M31* $\left(L_{5 \mathrm{GHz}} \sim 2 \times 10^{32} \mathrm{erg} \mathrm{s}^{-1}\right.$; Garcia et al. 2010). On the other hand, the measured value of $\Gamma(\sim 2.4)$ for X1 would be compatible with the empirical anti-correlation between the photon-index and the Eddington ratio in LLAGNs (Gu \& Cao 2009), for $L_{\mathrm{bol}} / L_{\mathrm{Edd}} \sim 10^{-7.5}$ of the SMBH in M32. This supports X1 being the genuine X-ray counterpart of the SMBH.

Now, assuming that both $\mathrm{R} 1$ and $\mathrm{X} 1$ are the manifestations of the central SMBH, it would be interesting to confront the radio and X-ray measurements with the FP. The "classical" FP (Equation 2) predicts $L_{\mathrm{R}} \sim 8.1 \times 10^{33} \mathrm{erg} \mathrm{s}^{-1}$ (for $M_{\mathrm{BH}} \approx 2.5 \times 10^{6} \mathrm{M}_{\odot}$ and $L_{\mathrm{X}} \approx 1.0 \times 10^{36}$ $\operatorname{erg~s}^{-1}$ ). Alternatively, the modified FP proposed by Yuan \& Cui (2005) and Yuan et al. (2009; Equation 5 therein),

$$
\log L_{\mathrm{R}}=1.29( \pm 0.03) \log L_{\mathrm{X}}+0.11( \pm 0.04) \log M_{\mathrm{BH}}-14.1
$$

which should operate at the low-luminosity regime $\left(L_{\mathrm{X}} \lesssim 10^{-6} L_{\mathrm{Edd}}\right)$ where the X-ray emission might be dominated by a synchrotron-cooled jet, predicts $L_{\mathrm{R}} \sim 1.1 \times 10^{33} \mathrm{erg} \mathrm{s}^{-1}$. Taking into

\footnotetext{
${ }^{5}$ Scaled from the average $0.5-10 \mathrm{keV}$ luminosity assuming the fiducial power-law spectrum (Section 3.2).
} 
account the scatter $(\sim 1.0$ dex; Gültekin et al. 2009) in the FPs, the predicted values are not inconsistent with the observed luminosity $\left(2.3 \times 10^{32} \mathrm{erg} \mathrm{s}^{-1}\right)$. This can be further considered a supporting evidence that both $\mathrm{R} 1$ and $\mathrm{X} 1$ are indeed physically related to the central SMBH. In this case, the slightly extended morphology of R1 might trace pc-scale jets/lobes or a core-plus-jets system.

In summary, our sensitive VLA observations have revealed a weak radio source coincident with the nucleus of M32. While at this stage a stellar origin cannot be completely ruled out for this nuclear source, we favor the interpretation that it is the long-sought radio counterpart of the central $\mathrm{SMBH}$, and suggest to designate it M32* for future reference, following the convention of Sgr A*, the radio counterpart of the Galactic Center Black Hole. Future VLA observations in multi-wavelength and with higher angular resolutions will be useful to unambiguously determine the nature of this source. Such observations will also benefit an in-depth investigation of the serendipitously detected radio $\mathrm{PNe}$ in the vicinity of $\mathrm{M} 32^{*}$.

The National Radio Astronomy Observatory is a facility of the National Science Foundation operated under cooperative agreement by Associated Universities, Inc. We thank M. Sarzi for valuable comments on the SAURON observations of PNe in M32. This work was partially supported by the National Natural Science Foundation of China (grants 11473010 and 11133001). Y.Y. is indebted to the hospitality of NRAO during her visit. Z.L. acknowledges support from the Recruitment Program of Global Youth Experts.

\section{REFERENCES}

Baganoff, F. K., Bautz, M. W., Brandt, W. N., et al. 2001, Nature, 413, 45

Baganoff, F. K., Maeda, Y., Morris, M., et al. 2003, ApJ, 591, 891

Bender, R., Kormendy, J., Bower, G., et al. 2005, ApJ, 631, 280

Brown, T. M., Bowers, C. W., Kimble, R. A., Sweigart, A. V., \& Ferguson, H. C. 2000, ApJ, 532, 308

Caplan, J., \& Deharveng, L. 1986, A\&A, 155, 297

Cash, W., Charles, P., \& Bowyer, S. 1979, A\&A, 72, L6

Davies, R. D., Walsh, D., \& Booth, R. S. 1976, MNRAS, 177, 319

Falcke, H., Körding, E., \& Markoff, S. 2004, A\&A, 414, 895

Gallo, E., Treu, T., Marshall, P. J., et al. 2010, ApJ, 714, 25

Garcia, M. R., Hextall, R., Baganoff, F. K., et al. 2010, ApJ, 710, 755 


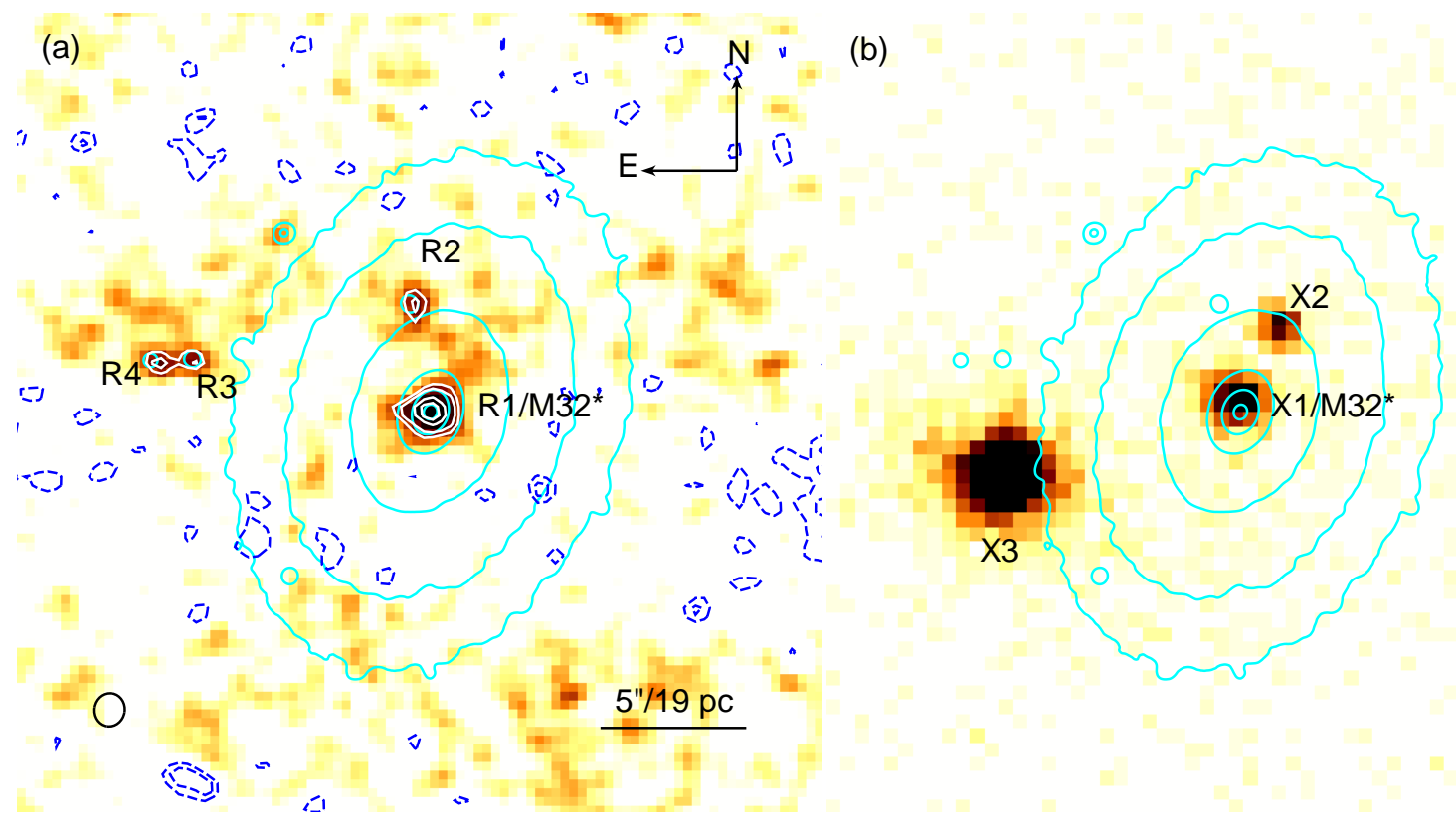

Fig. 1.- (a) The 18-hr integrated VLA $C$-band image of the central $25^{\prime \prime} \times 25^{\prime \prime}(\sim 95$ pc $\times 95$ pc) region of M32, overlaid by intensity contours (positive in white solid and negative in blue dashed) at levels of $-3,-2,4,5,7$ and 8 times the average rms $\left(1.2 \mu \mathrm{Jy}_{\text {beam }}{ }^{-1}\right)$. Also shown are HST/WFC3/F502N intensity contours (cyan) highlighting the starlight distribution of M32. Four compact sources are labeled, among which R1 is coincident with the nucleus of M32 (named M32*), while R2, R3 and R4 are coincident with optical PNe. The black ellipse on the lower left-hand corner represents the synthesized beam size. (b) A Chandra 0.3-8 keV image showing the same region, overlaid by the F502N intensity contours. The three X-ray sources are labeled, following the convention of HTU03. 


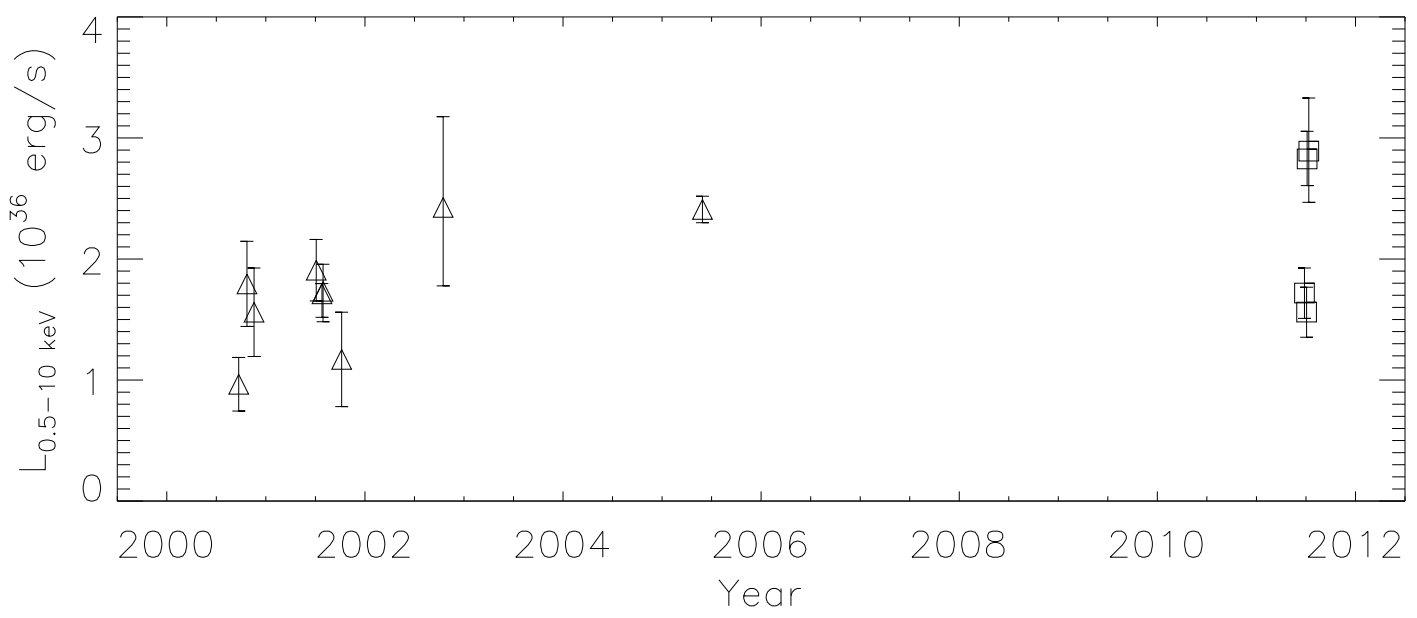

Fig. 2.- A long-term 0.5-10 keV light curve of X1 based on nine Chandra (triangles) and four $X M M-N e w t o n$ (squares) observations. See text for details.

Table 1. X-ray observations of M32

\begin{tabular}{cccc}
\hline \hline $\begin{array}{c}\text { ObsID } \\
(1)\end{array}$ & $\begin{array}{c}\text { Date } \\
(2)\end{array}$ & $\begin{array}{c}\text { Exposure } \\
(3)\end{array}$ & $\begin{array}{c}L_{\mathrm{X}} \\
(6)\end{array}$ \\
\hline 313 (C/ACIS-S) & $2000-09-21$ & 6.0 & $1.0_{-0.2}^{+0.2}$ \\
314 (C/ACIS-S) & $2000-10-21$ & 5.1 & $1.8_{-0.4}^{+0.4}$ \\
1580 (C/ACIS-S) & $2000-11-17$ & 5.1 & $1.6_{-0.4}^{+0.4}$ \\
1584 (C/ACIS-I) & $2001-07-03$ & 4.9 & $1.9_{-0.3}^{+0.3}$ \\
2017 (C/ACIS-S) & $2001-07-24$ & 45.9 & $1.7_{-0.2}^{+0.2}$ \\
2494 (C/ACIS-S) & $2001-07-28$ & 16.0 & $1.7_{-0.3}^{+0.3}$ \\
1576 (C/ACIS-I) & $2001-10-05$ & 4.9 & $1.2_{-0.4}^{+0.4}$ \\
2894 (C/ACIS-I) & $2002-10-14$ & 4.7 & $2.4_{-0.8}^{+0.7}$ \\
5690 (C/ACIS-S) & $2005-05-27$ & 113.0 & $2.4_{-0.3}^{+0.3}$ \\
0672130101 (X) & $2011-06-27$ & 99.4 & $1.9_{-0.3}^{+0.3}$ \\
0672130601 (X) & $2011-07-05$ & 119.9 & $1.7_{-0.3}^{+0.3}$ \\
0672130701 (X) & $2011-07-07$ & 101.7 & $3.2_{-0.4}^{+0.4}$ \\
0672130501 (X) & $2011-07-13$ & 55.8 & $2.8_{-0.4}^{+0.4}$ \\
\hline
\end{tabular}

Note. - (1) Observation ID. Chandra observations are denoted by "C/ACIS-S" or "C/ACIS-I" and XMMNewton observations by "X"; (2) Date of observation; (3) Effective exposure, in ks; (6) $0.5-10 \mathrm{keV}$ unabsorbed luminosity of the nuclear source, in units of $10^{36} \mathrm{erg} \mathrm{s}^{-1}$. 
Genzel, R., Eisenhauer, F., \& Gillessen, S. 2010, Reviews of Modern Physics, 82, 3121

Ghez, A. M., Salim, S., Weinberg, N. N., et al. 2008, ApJ, 689, 1044

Gültekin, K., Cackett, E. M., Miller, J. M., et al. 2009, ApJ, 706, 404

Gu, M., \& Cao, X. 2009, MNRAS, 399, 349

Ho, L. C., Filippenko, A. V., \& Sargent, W. L. W. 1997, ApJS, 112, 315

Ho, L. C., \& Ulvestad, J. S. 2001, ApJS, 133, 77

Ho, L. C., Filippenko, A. V., \& Sargent, W. L. W. 2003, ApJ, 583, 159

Ho, L. C., Terashima, Y., \& Ulvestad, J. S. 2003, ApJ, 589, 783 (HTU03)

Hyman, S. D., Lazio, T. J. W., Kassim, N. E., \& Bartleson, A. L. 2002, AJ, 123, 1497

Hyman, S. D., Wijnands, R., Lazio, T. J. W., et al. 2009, ApJ, 696, 280

Jarrett, T. H., Chester, T., Cutri, R., Schneider, S. E., \& Huchra, J. P. 2003, AJ, 125, 525

Kaur, A., Henze, M., Haberl, F., et al. 2012, American Astronomical Society Meeting Abstracts \#219, 219, \#442.05

Kowk, S. 2000, The origin and evolution of planetary nebula, New York, Cambridge University Press

Li, Z., Garcia, M. R., Forman, W. R., et al. 2011, ApJ, 728, L10

Marconi, A., Risaliti, G., Gilli, R., et al. 2004, The Interplay Among Black Holes, Stars and ISM in Galactic Nuclei, 222, 49

Merloni, A., Heinz, S., \& di Matteo, T. 2003, MNRAS, 345, 1057

Miller, B., Gallo, E., Treu, T., \& Woo, J.-H. 2012, ApJ, 745, LL13

Muno, M. P., Pfahl, E., Baganoff, F. K., et al. 2005, ApJ, 622, L113

Nagar, N. M., Falcke, H., Wilson, A. S., \& Ho, L. C. 2000, ApJ, 542, 186

Narayan, R., \& Yi, I. 1994, ApJ, 428, L13

Perley, R. A., \& Butler, B. J. 2013, ApJS, 204, 19

Remillard, R. A., \& McClintock, J. E. 2006, ARA\&A, 44, 49

Revnivtsev, M., Churazov, E., Sazonov, S., Forman, W., \& Jones, C. 2007, A\&A, 473, 783

Sage, L. J., Welch, G. A., \& Mitchell, G. F. 1998, ApJ, 507, 726 
Sarzi M., Mamon, G. A., Cappellari, M., Emsellem, E., Bacon, R., Davies, R., \& de Zeeuw, P. T. 2011, MNRAS, 415, 2832

Seth, A. C. 2010, ApJ, 725, 670

Soltan, A. 1982, MNRAS, 200, 115

Verolme, E. K., Cappellari, M., Copin, Y., et al. 2002, MNRAS, 335, 517

van den Bosch, R. C. E., \& de Zeeuw, P. T. 2010, MNRAS, 401, 1770

Welch, G. A., \& Sage, L. J. 2001, ApJ, 557, 671

Wu, Q., \& Gu, M. 2008, ApJ, 682, 212

Young, C. K.-s., Currie, M. J., Dickens, R. J., Luo, A.-L., \& Zhang, T.-J. 2008, Chinese J. Astron. Astrophys., 8, 369

Yuan, F., Yu, Z., \& Ho, L. C. 2009, ApJ, 703, 1034

Yu, Q., \& Tremaine, S. 2002, MNRAS, 335, 965

Yuan, F., \& Cui, W. 2005, ApJ, 629, 408

Yuan, F., \& Narayan, R. 2014, ARA\&A, 52, 529

Zhao, J.-H., Roberts, D. A., Goss, W. M., et al. 1992, Science, 255, 1538

Zhao, J.-H., Bower, G. C., \& Goss, W. M. 2001, ApJ, 547, L29

Zijlstra, A. A., van Hoof, P. A. M., \& Perley, R. A. 2008, ApJ, 681, 1296 
Table 2. Properties of detected radio sources

\begin{tabular}{ccccccc}
\hline \hline $\begin{array}{c}\text { Source } \\
(1)\end{array}$ & $\begin{array}{c}\mathrm{RA} \\
(2)\end{array}$ & $\begin{array}{c}\mathrm{DEC} \\
(3)\end{array}$ & $\begin{array}{c}I_{\mathrm{p}} \\
(4)\end{array}$ & $\begin{array}{c}S_{6.6} \\
(5)\end{array}$ & $\begin{array}{c}L_{6.6} \\
(6)\end{array}$ & $\begin{array}{c}L_{[\mathrm{OIII}]} \\
(7)\end{array}$ \\
\hline R1 & $00^{h} 42^{m} 41^{\mathrm{s}} .838$ & $+40^{\circ} 51^{\prime} 54^{\prime \prime} .98$ & $10.7 \pm 1.2$ & $47.3 \pm 6.1$ & 22.7 & $<4.3$ \\
$\mathrm{R} 2$ & $00^{h} 42^{m} 41^{\mathrm{s}} .891$ & $+40^{\circ} 51^{\prime} 58^{\prime \prime} .41$ & $6.6 \pm 1.2$ & $3.5 \pm 1.2$ & 1.7 & 11.1 \\
$\mathrm{R} 3$ & $00^{h} 42^{m} 42^{\mathrm{s}} .563$ & $+40^{\circ} 51^{\prime} 56^{\prime \prime} .52$ & $6.6 \pm 1.2$ & $3.4 \pm 1.2$ & 1.6 & 7.0 \\
$\mathrm{R} 4$ & $00^{h} 42^{m} 42^{\mathrm{s}} .659$ & $+40^{\circ} 51^{\prime} 56^{\prime \prime} .41$ & $6.8 \pm 1.2$ & $3.5 \pm 1.2$ & 1.7 & 5.6 \\
\hline
\end{tabular}

Note. - (1) Source name; (2)-(3) Source position in Celestial coordinates; (4) Peak flux density, in units of $\mu \mathrm{Jy}_{\text {beam }}{ }^{-1}$; (5) Total integrated flux density and error at 6.6 $\mathrm{GHz}$, in units of $\mu \mathrm{Jy}$; (6) Monochromatic luminosity at $6.6 \mathrm{GHz}$, in units of $10^{31} \mathrm{erg} \mathrm{s}^{-1}$; (7) $[\mathrm{O}$ III $] \lambda 5007$ emission line luminosity (or $3 \sigma$ upper limit), in units of $10^{35} \mathrm{erg} \mathrm{s}^{-1}$, from Sarzi et al. (2011). 